\title{
Polymorph Evolution of Organic Molecules during Crystal Growth Studied by MicroED
}

\author{
M Lightowler ${ }^{1}, \mathrm{E}$ Broadhurst ${ }^{2}, \mathrm{H} \mathrm{Xu^{3 } , \mathrm { M } \mathrm { Clabbers } ^ { 4 } , \mathrm { F } \text { Nudelman } ^ { 5 } , \mathrm { X } \mathrm { Zou } ^ { 6 } \text { , S Parsons }}{ }^{5}$ \\ ${ }^{1}$ Stockholm University, Stockholm, ${ }^{2}$ The University of Edinburgh, Edinburgh, United Kingdom, \\ ${ }^{3}$ Department of Materials and Environmental Chemistry, Stockholm, Sweden, ${ }^{4}$ Stockholm \\ University, Djursholm, ${ }^{5}$ The University of Edinburgh, Edinburgh, -- SELECT --, ${ }^{6}$ Department of \\ Materials and Environmental Chemistry, Stockholm, -- SELECT -- \\ molly.lightowler@mmk.su.se
}

Rapid, atomic resolution structure determination of nanometre-sized crystals of small organic molecules can be obtained using MicroED. In this work, we combined the capabilities of MicroED for the study of very small crystallites with [i]in situ[/i] crystal growth to follow the sequential polymorph evolution of glycine from an aqueous solution ([i]1[/i]). The three known polymorphs of glycine which exist under ambient conditions follow the stability order $\beta<\alpha<\gamma$. Crystal growth in droplets of the glycine solution $(3 \mu \mathrm{L})$ was stopped by solvent removal and plunge freezing after 3, 4 and 5 minutes. The ability to observe the crystallization process in timeslices of only one minute enabled the fast, dynamic process to be observed at shorter timescales than has previously been possible. The thermodynamically least stable $\beta$ polymorph forms exclusively after 3 minutes, but this begins to yield to the $\alpha$ form after just one minute more. One crystallite of the $\gamma$ form was observed after the drop was allowed to evaporate to dryness over the course of 1 hour. Using this gentle and non-invasive technique, we could rapidly identify and selectively determine the crystal structures of all three polymorphic forms from a single sample, despite their presence in vanishingly small quantities.

In Zou's lab, we have developed serial electron diffraction (SerialED) to automate data collection by taking electron diffraction patterns of individual particles ([i]2[/i]). Diffraction data from up to 3500 crystals per hour can be collected on a standard TEM. We have also combined data collection by SerialED with rotation electron diffraction (RED) and developed SerialRED to perform fully automated data collection and data analysis for 3D electron diffraction ([i]3[/i]). This provides new possibilities for studying beam-sensitive crystals, quantitative phase analysis and detection of minor phases in the sample. These methods, along with [i]in situ[/i] crystal growth, can greatly accelerate polymorphic discovery and provide new possibilities to study reaction mixtures, dynamical processes and other applications where specific solid forms of materials are required.

1. E. Broadhurst, H. Xu, M. Clabbers, M. Lightowler, F. Nudelman, X. Zou, S.Parsons, [i]IUCrJ[/i] [b]7[/b], 5 (2020).

2. S. Smeets, X. Zou, W. Wan, [i]J. Appl. Cryst.[/i] [b]51[/b], 1262 (2018).

3. B. Wang, X. Zou, S. Smeets, [i]IUCrJ[/i] [b]6[/b], 854 (2019).

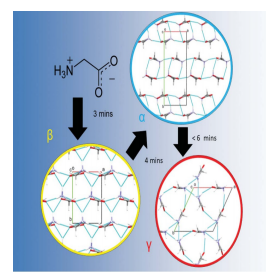

Figure 1

Acta Cryst. (2020). A76, a154 